\section{RMD Open}

Rheumatic \&

Musculoskeletal Diseases

\title{
Fully automated joint space width measurement and digital X-ray radiogrammetry in early $\mathrm{RA}$
}

Michael Platten, ${ }^{1}$ Yogan Kisten, ${ }^{1}$ Johan Kälvesten, ${ }^{2,3}$ Laurent Arnaud, ${ }^{1}$ Kristina Forslind, ${ }^{4,5}$ Ronald van Vollenhoven ${ }^{1,6}$

To cite: Platten M, Kisten Y, Kälvesten J, et al. Fully automated joint space width measurement and digital $X$-ray radiogrammetry in early RA. RMD Open 2017;3:e000369. doi:10.1136/ rmdopen-2016-000369

- Prepublication history and additional material are available. To view these files please visit the journal online (http://dx.doi.org/ 10.1136/ rmdopen-2016-000369).

Received 26 September 2016 Revised 8 April 2017 Accepted 16 April 2017

\section{CrossMark}

For numbered affiliations see end of article.

Correspondence to Michael Platten; michael. platten@stud.ki.se

\section{ABSTRACT}

Objectives To study fully automated digital joint space width (JSW) and bone mineral density (BMD) in relation to a conventional radiographic scoring method in early rheumatoid arthritis (eRA).

Methods Radiographs scored by the modified Sharp van der Heijde score (SHS) in patients with eRA were acquired from the SWEdish FarmacOTherapy study. Fully automated JSW measurements of bilateral metacarpals 2, 3 and 4 were compared with the joint space narrowing (JSN) score in SHS. Multilevel mixed model statistics were applied to calculate the significance of the association between $\triangle \mathrm{JSW}$ and $\triangle \mathrm{BMD}$ over 1 year, and the JSW differences between damaged and undamaged joints as evaluated by the JSN.

Results Based on 576 joints of 96 patients with eRA, a significant reduction from baseline to 1 year was observed in the JSW from $1.69( \pm 0.19) \mathrm{mm}$ to 1.66 $( \pm 0.19) \mathrm{mm}(\mathrm{p}<0.01)$, and BMD from $0.583( \pm 0.068)$ $\mathrm{g} / \mathrm{cm}^{2}$ to $0.566( \pm 0.074) \mathrm{g} / \mathrm{cm}^{2}(\mathrm{p}<0.01)$. A significant positive association was observed between $\triangle \mathrm{JSW}$ and $\triangle B M D$ over 1 year $(p<0.0001)$. On an individual joint level, JSWs of undamaged (JSN=0) joints were wider than damaged (JSN>0) joints: $1.68 \mathrm{~mm}(95 \% \mathrm{Cl} 1.70$ to 1.67$)$ vs $1.54 \mathrm{~mm}(95 \% \mathrm{Cl} 1.63$ to 1.46$)$. Similarly the unadjusted multilevel model showed significant differences in JSW between undamaged $(1.68 \mathrm{~mm}$ $(95 \% \mathrm{Cl} 1.72$ to 1.64$))$ and damaged joints $(1.63 \mathrm{~mm}$ (95\% Cl 1.68 to 1.58$))(p=0.0048)$. This difference remained significant in the adjusted model: $1.66 \mathrm{~mm}$ (95\% Cl 1.70 to 1.61$)$ vs $1.62 \mathrm{~mm}(95 \% \mathrm{Cl} 1.68$ to 1.56$)$ $(p=0.042)$.

Conclusions To measure the JSW with this fully automated digital tool may be useful as a quick and observer-independent application for evaluating cartilage damage in eRA.

Trial registration number NCT00764725.

\section{INTRODUCTION}

Despite technological advancements and the availability of ultrasound (US) and MRI modalities, conventional radiography (CR) remains the main imaging tool for rheumatoid arthritis (RA). The clinical use of radiographs as permanent medical records

\section{Key messages}

What is already known about this subject?

- Cartilage damage, measured as joint space narrowing (JSN), negatively affects the functional status of patients with rheumatoid arthritis (RA), and lately some studies have used digital radiography programs to detect the chronological change in joint space width (JSW) between baseline and follow-up images.

What does this study add?

- This study describes a fully automated JSW program that takes advantage of the knowledge of the type of joint being evaluated by measuring the joint radially over an angular width of $3 \pi / 8$ of the metacarpal head surface, resulting in a low coefficient of variation.

- The program can detect subtle changes in JSW and differentiate between damaged and undamaged joints, and this change in JSW correlates well with the visual JSN of the modified Sharp van der Heijde method.

- Knowledge that in early RA, the loss of cartilage as measured by JSW and demineralisation of bone as measured by bone mineral density (BMD) loss take place concurrently and are associated with each other.

How might this impact on clinical practice?

- Fully automated programs measuring JSW and BMD have the potential to make this information more readily available and thus more feasible for integration into clinical practice.

has several strengths, that is, the technology is accessible globally, they may be evaluated at any time, the severity of structural damage and progression can be assessed, and treatment effects determined. ${ }^{1}$ RA inflammation has a predilection for small joint involvement, and radiographs of the metacarpophalangeal (MCP) and metatarsophalangeal joints display radiographic progression particularly well. $^{2}$ Joint space narrowing (JSN) due to destroyed cartilage may have a larger effect 
on functional status than erosions, making it a valid target for treatment. ${ }^{3}$

In RA clinical trials, the van der Heijde modification of the Sharp score (SHS) ${ }^{4}$ is currently the most frequently used scoring method for evaluating radiographic progression with JSN and erosions of selected joints in hands and feet. Although this scoring method has many strengths, its weaknesses are that the scoring is time-consuming and that it requires specialised training that seldom is available to the rheumatologists. ${ }^{5}$ In order to minimise the aforementioned limitations, semiautomated and fully automated software to better evaluate joint space width (JSW) have been developed and reviewed.$^{6-10}$ The benefits of a fully automated program include higher reproducibility, faster results, observer independence and a higher sensitivity. ${ }^{11-13}$ Moreover, bone mineral density (BMD) measured with digital X-ray radiogrammetry (DXR) is a recent tool that quantifies BMD in metacarpals 2, 3 and $4 .{ }^{14}$ DXR has been proven sensitive in measuring BMD loss, ${ }^{15}$ and BMD loss has been shown to be associated with an increased risk of radiographic progression. ${ }^{16}{ }^{17}$ Rapid loss of BMD is predictive of radiographic progression, ${ }^{17} 18$ and this has been shown already after 4 months. ${ }^{19}$ Using the SWEdish FarmacOTherapy (SWEFOT) study data of patients with early RA, we now investigate how JSW compares with JSN and explore the association between BMD and JSW using a multilevel mixed model, which has not previously been done. We also discuss the utility of these new tools in clinical practice.

\section{METHODS}

\section{Patients and study design}

Data of patients with early RA were acquired from the SWEFOT study. The detailed study design has previously been reported ${ }^{20}$ and is summarised in the online supplementary material. Of the 487 patients in the SWEFOT study, 119 patients met the inclusion criteria for this study (figure 1) and constitute the patients in the multilevel mixed model. Furthermore, 96 patients had both baseline and follow-up radiographs, which allowed for comparisons of progress over 12 months. A flow chart illustrating the selection process is shown in figure 1.

\section{Radiographic assessment}

Radiographs of the hands and feet were obtained at baseline and 1 year.

\section{Radiographic positioning of the hand}

Radiographic examinations were performed using a posteroanterior (PA) projection of the hand. The PA projection is the best conventional view for demonstrating malalignment, JSN and soft-tissue abnormalities in early RA. Bilateral hands are generally X-rayed, with the contralateral image used for bony structure comparison.

\section{Technical factors}

An image receptor (IR), $10 \times 12$ inch $(24 \times 30 \mathrm{~cm})$ crosswise for two or more images on one cassette, was used. A digital screen, lead masking and collimation is used for radiation protection and avoidance of scatter. A range of $50-60 \mathrm{kVp}$ and 3-4 mAs were used, as well as a minimum source to image distance (SID) of $100 \mathrm{~cm}$.

\section{Positioning for PA projection}

First the patient is asked to sit at the end of the radiographic table that is adjusted to the patient's height so that the forearm is resting on the table. Second, the patient's forearm is well rested on the table with the hands placed with the palmar surface flat onto the cassette. Then the radiographic plate/cassette is centred to the MCP joints, and adjusted to the long axis of the cassette parallel with the long axis of the hand and forearm. Then the patient is asked to spread the fingers slightly to ensure correct positioning. The patient is then asked to relax the hand to avoid motion. Adhesive tape or positioning sponges can be used to prevent involuntary movement. A sandbag may be placed over the distal forearm if necessary. Finally,

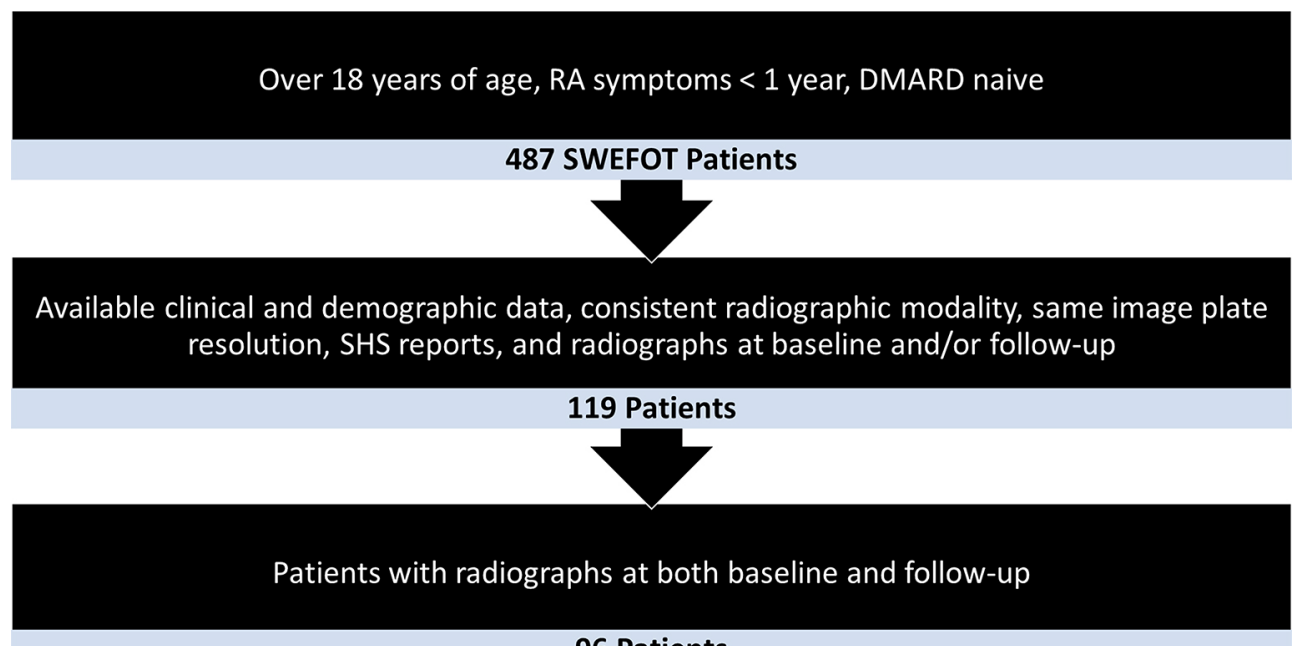

96 Patients

Figure 1 Flow chart of patient selection. 
a central ray is directed so that it is perpendicular to the third MCP joint.

\section{Evaluation criteria}

First, all fingers, the wrist and about $2.5 \mathrm{~cm}$ of the distal forearm should be visible. Second the MCP and interphalangeal joints (clear joint spaces) should appear open, indicating correct central ray location and that the hand was fully pronated. The long axis of the IR should be well aligned to the long axis of the hand and wrist. Third, free from positioning error, both sides of the hands should appear symmetrical unless pathology is suspected with no evidence of rotational error. The concavities of the shafts of the metacarpals and the phalanges $2-5$, and the amount of soft tissue on either side of phalanges 2-5, should appear equal unless pathology is suspected. The digits should not overlap, and the fingers should be separated slightly with soft tissues clearly delineated.

These images of the hands, per standardised radiography protocol, are the ones used for radiographic assessment by both the clinician and the fully automated JSW program. Only images using the same modality type and settings at baseline and follow-up were analysed. Radiographic joint damage was assessed according to the $\mathrm{SHS}^{4}$ method, by visual inspection of the radiographs and using a semiquantitative scale of $0-4$ for JSN. The radiographs were read in chronological order by one of two experienced readers blinded to clinical data. The interclass correlation coefficient was 0.94 and the smallest detectable difference (SDD) in SHS was 5.8 units. ${ }^{16}$

\section{Fully automated JSW measurements}

Computer-assisted automated measurements of the MCP joint spaces were calculated from the digital hand radiographs of each patient using dedicated software (dxr-online, Sectra, Linköping, Sweden). This software was a further development of a semiautomated version used previously. ${ }^{1321-23}$ The detailed method for measuring JSW is described in the online supplementary material. The measurement regions are illustrated in figure 2. The short-term in vivo reproducibility of JSW was tested with 30 healthy individuals using the same machine, radiographer and protocol at three separate time points on the same day. The hand was repositioned, that is, moved, between the three images. This was expressed as the coefficient of variation (CV\%), which was $1.4 \%$ and the SDD was $0.062 \mathrm{~mm}$ for MCP 2, 3 and 4 of the 30 healthy volunteers.

\section{Metacarpal BMD}

In the DXR-BMD method, the narrowest part of the metacarpals 2, 3 and 4 is located. The cortical thickness and bone width are measured within the measurement regions. By assuming that bone density is constant and the bone elliptical, the BMD can be calculated in $\mathrm{g} /$ $\mathrm{cm}^{2} .{ }^{24}$ The measurement regions are shown in figure 2 .

\section{Statistics}

Normal distribution was tested using the Shapiro-Wilk test. For unpaired data, comparison between groups was

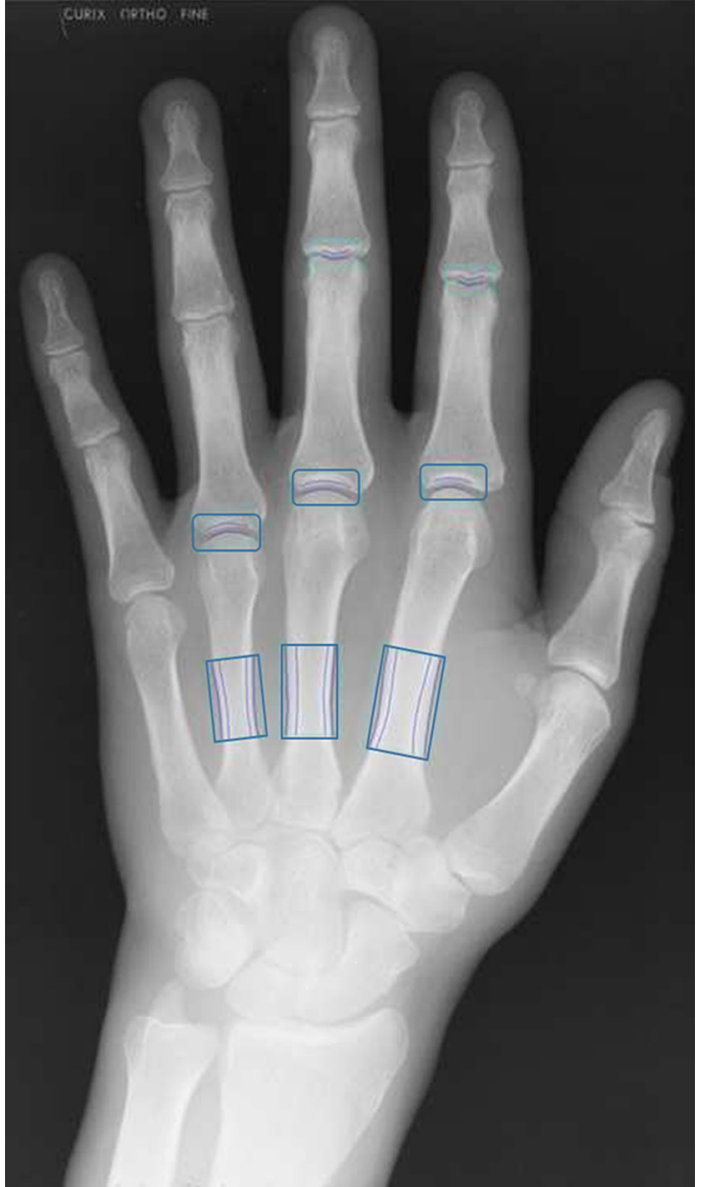

Figure 2 Radiograph of hand with highlighted measurement regions. Posteroanterior radiograph of the left hand with measurement boxes placed at MCP 2, 3 and 4 in order to measure joint space width. Measurement boxes are also placed on the metacarpal bones 2, 3 and 4 in order to measure bone mineral density. MCP, metacarpophalangeal.

performed using the unpaired t-test for normally distributed data, and the Mann-Whitney test for non-normally distributed data. For paired data, we used the paired t-test in case of normally distributed data, and the Wilcoxon test for non-normally distributed data, to determine whether the change over 12 months was significant. A multilevel mixed model was used to explore if there was a significant association between $\triangle \mathrm{JSW}$ and $\triangle \mathrm{BMD}$ over 1 year. This model clustered $\triangle \mathrm{JSW}$ and $\triangle \mathrm{BMD}$ on patient level and each hand separately. It also took into account repeated visits, that is, baseline and 1 year follow-up, and adjusted for height, age, gender and BMI. Additional multilevel mixed models were used to establish the difference in JSW between individual joints with cartilage damage (JSN score $>0$ ) versus joints with no cartilage damage (JSN score $=0$ ). These multilevel models took into account the correlations between the joints from the same hand as well as with the contralateral joints, since these joints are intrinsically related to each other. In these models, JSW was considered the dependent variable, and cartilage damage in the form of JSN the explanatory variable. We constructed models taking into account baseline and 
Table 1 Demographic data of the whole SWEFOT population versus the study cohort

\begin{tabular}{llll}
\hline Demographic & SWEFOT patients $(\mathbf{n = 4 8 7})$ & Study patients $(\mathbf{n = 1 1 9 )}$ & $\mathbf{p ~ V a l u e ~}$ \\
\hline Female sex, $\mathrm{n}(\%)$ & $344(70.6)$ & $93(78.2)$ & 0.13 \\
ACPA pos, $\mathrm{n}(\%)$ & $310(63.7)$ & $60(57.7)$ & 0.25 \\
RF pos, $\mathrm{n}(\%)$ & $333(68.4)$ & $76(65.0)$ & 0.47 \\
DAS28, mean (SD) & $5.73(1.01)$ & $5.64(0.95)$ & 0.39 \\
ESR (mm), mean (SD) & $39.8(28.1)$ & $37.5(24.3)$ & 0.45 \\
CRP (mg/L), mean (SD) & $33.7(42.4)$ & $34.8(38.1)$ & 0.69 \\
HAQ, mean (SD) & $1.19(0.58)$ & $1.18(0.56)$ & 0.95 \\
SHS, mean (SD) & $4.54(8.01)$ & $4.49(8.20)$ & 0.74 \\
JSN, mean (SD) & $2.63(7.08)$ & $2.45(5.68)$ & 0.50 \\
Erosion score, mean (SD) & $2.03(3.70)$ & 0.34 \\
\hline
\end{tabular}

ACPA, anticitrullinated peptide antibody; CRP, C reactive protein; DAS28, Disease Activity Score of 28 joints; ESR, erythrocyte sedimentation rate; HAQ, Health Assessment Questionnaire; JSN, joint space narrowing; Pos, positive; RF, rheumatoid factor; SHS, modified Sharp van der Heijde Score; SWEFOT, SWEdish FarmacOTherapy.

follow-up visits as repeated measures. These models were further adjusted for height, age, gender and BMI, which have been shown to influence JSW. ${ }^{22} 25$

All tests were bilateral and $p$ values of $<0.05$ were considered statistically significant. The statistical analyses were performed with SPSS Statistics V.23, Excel 2013 V.15.0 (Microsoft, Redmond, Washington, USA) and Prism V.6.07 for Windows (GraphPad Software, La Jolla, California, USA).

\section{Ethical considerations}

All patients gave their informed consent and the study was performed in accordance with the Declaration of Helsinki. Ethical approval for the present study was obtained from the ethical board in all participating units and was registered at http://www.clinicaltrials.gov (NCT00764725).

\section{RESULTS}

\section{Baseline patient characteristics}

A total of 119 patients with early RA, 78\% female and with a mean age of $53( \pm 14.4)$ years, were included in this study. Baseline characteristics are shown in table 1, where they are compared with the whole SWEFOT population.

\section{Assessment of JSW}

The change of JSW in both hands from baseline to 1 year is shown in table 2 and figure $3 \mathrm{~A}$. There is a significant decrease over 12 months in the mean JSW of the two

\begin{tabular}{|c|c|c|c|}
\hline Measure $(m e a n \pm S D)$ & Baseline $(n=96)$ & 12 Month follow-up ( $n=96)$ & p Value \\
\hline Left hand BMD $\left(\mathrm{g} / \mathrm{cm}^{2}\right)$ & $0.577 \pm 0.069$ & $0.561 \pm 0.074$ & $<0.01$ \\
\hline Left hand JSW (mm) & $1.68 \pm 0.20$ & $1.66 \pm 0.21$ & 0.11 \\
\hline MCP $2(\mathrm{~mm})$ & $1.83 \pm 0.23$ & $1.81 \pm 0.22$ & 0.15 \\
\hline MCP $3(\mathrm{~mm})$ & $1.65 \pm 0.21$ & $1.63 \pm 0.23$ & 0.25 \\
\hline MCP $4(\mathrm{~mm})$ & $1.55 \pm 0.23$ & $1.54 \pm 0.24$ & 0.07 \\
\hline Right hand BMD (g/cm²) & $0.589 \pm 0.069$ & $0.572 \pm 0.075$ & $<0.01$ \\
\hline Right hand JSW (mm) & $1.70 \pm 0.19$ & $1.67 \pm 0.19$ & $<0.01$ \\
\hline MCP $2(\mathrm{~mm})$ & $1.86 \pm 0.23$ & $1.83 \pm 0.23$ & 0.03 \\
\hline MCP $3(\mathrm{~mm})$ & $1.66 \pm 0.20$ & $1.62 \pm 0.21$ & $<0.01$ \\
\hline MCP $4(\mathrm{~mm})$ & $1.58 \pm 0.23$ & $1.55 \pm 0.22$ & $<0.01$ \\
\hline Both hands JSW (mm) & $1.69 \pm 0.19$ & $1.66 \pm 0.19$ & $<0.01$ \\
\hline Both hands BMD $\left(\mathrm{g} / \mathrm{cm}^{2}\right)$ & $0.583 \pm 0.068$ & $0.566 \pm 0.074$ & $<0.01$ \\
\hline Erosion score & $1.72 \pm 3.26$ & $2.59 \pm 3.82$ & $<0.01$ \\
\hline JSN Score & $2.12 \pm 5.08$ & $3.89 \pm 6.05$ & $<0.01$ \\
\hline SHS & $3.84 \pm 7.35$ & $6.48 \pm 8.22$ & $<0.01$ \\
\hline
\end{tabular}

BMD, bone mineral density; JSN, joint space narrowing; JSW, joint space width; MCP, metacarpophalangeal; SHS, Sharp van der Heijde score. 


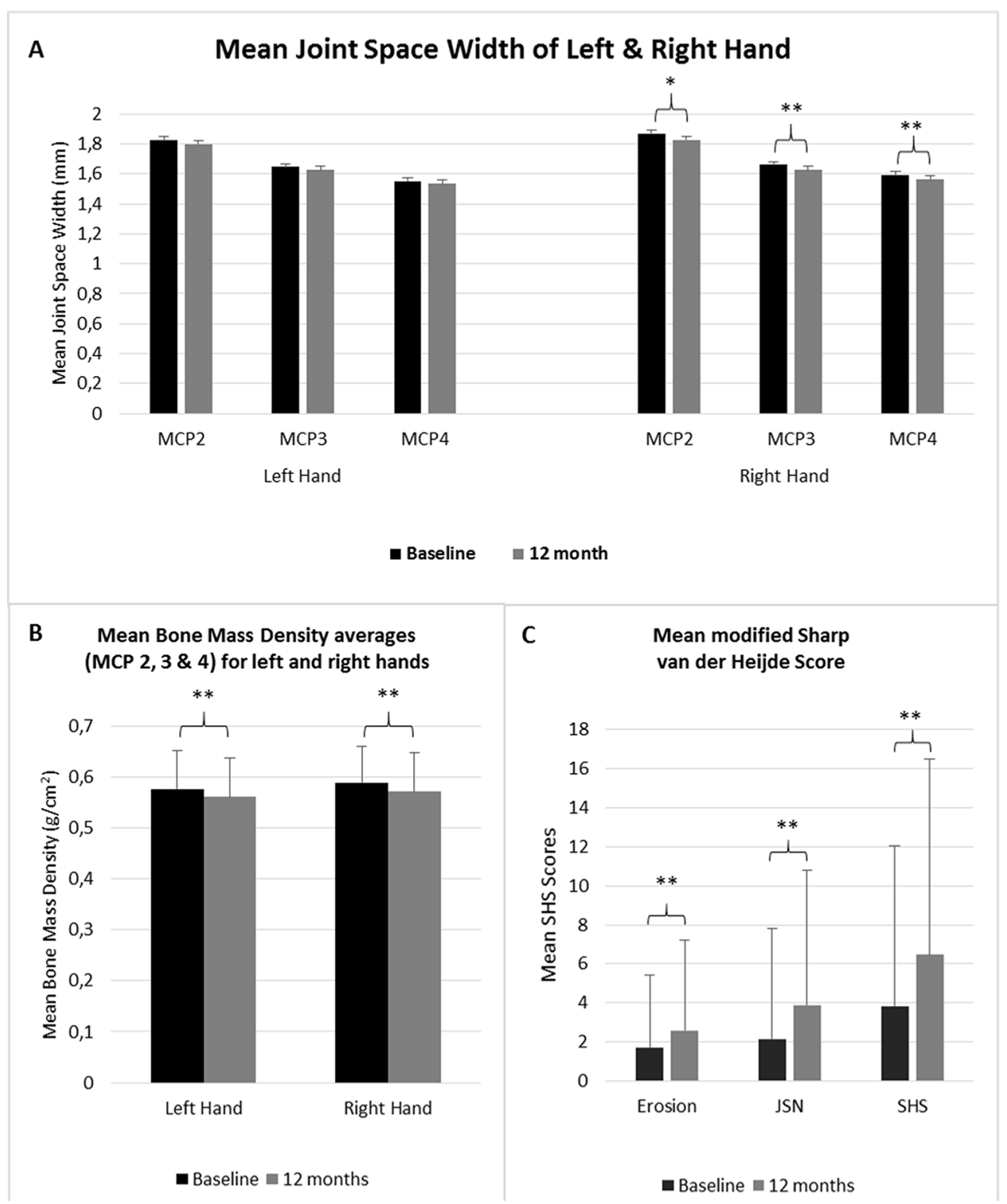

Figure 3 JSW, BMD and SHS at baseline and 12 months. Whiskers demonstrate SD of the mean. (A) Demonstrates the JSW of MCP 2, 3 and 4 at baseline and at 1 year. (B) Demonstrates the BMD of the left and right hand at baseline and at 1 year. (C) Demonstrates erosion score, JSN score and the SHS at baseline and at 1 year. *Significant difference, $p<0.05$; ${ }^{* *}$ significant difference, $\mathrm{p}<0.01$. BMD, bone mineral density; JSN, joint space narrowing; JSW, joint space width; MCP, metacarpophalangeal; SHS, Sharp van der Heijde score.

hands combined, as well as in the right hand. The left hand JSW revealed a decrease over 12 months, which was not statistically significant.

\section{Assessment of BMD}

The detailed baseline and 1 year follow-up BMDs are shown in table 2 and figure 3B. The mean BMD decreased significantly in both hands combined, as well as in the right and left hand separately.

\section{Assessment of modified SHS}

SHS and its components, JSN and erosion score, are shown from baseline to 1 year in table 2 and figure 3C. Both the JSN and erosion components increased significantly over 12 months.

\section{Multilevel mixed model}

The multilevel model clustering $\triangle \mathrm{JSW}$ and $\triangle \mathrm{BMD}$ showed a significant $(\mathrm{p}<0.0001)$ positive association from baseline to 1 year follow-up in the 96 patients. This model was adjusted for height, age, gender and BMI.

The JSW for joints with damage (JSN $>0$ ) and without damage (JSN=0) was compared using the raw data and a multilevel mixed model (figure 4). The raw data of the mean JSW at baseline and 12 months showed that the undamaged joints were wider than the damaged joints, $1.68 \mathrm{~mm}(95 \%$ CI 1.70 to 1.67$)$ and $1.54 \mathrm{~mm}$ (95\% CI 1.63 to 1.46$)$, respectively. This value, however, is not statistically significant as it does not account for repeated visits, that is, baseline and follow-up, and that the patient's own 
Distribution of Joints with Damage or No Damage

Raw Data, Unadjusted \& Adjusted Model

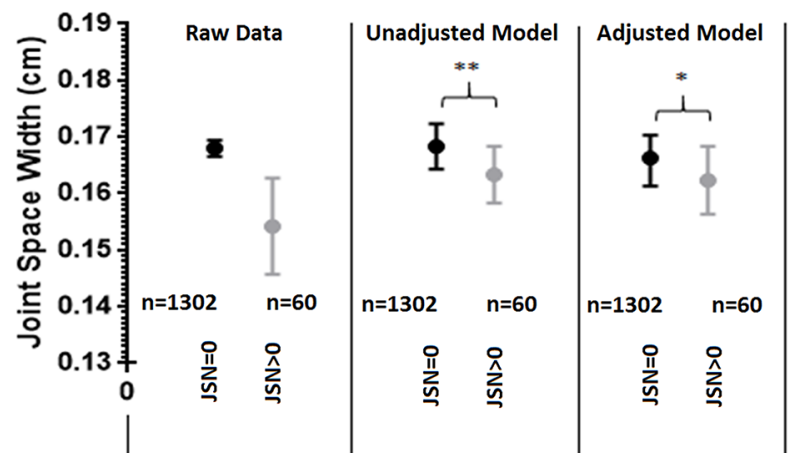

Figure 4 Distribution of joints with damage or no damage: raw data, unadjusted and adjusted model. On an individual joint level, the JSW when a joint received a JSN score of 0 and when a joint received a JSN score of $>0$ for the raw data, as well as for the unadjusted and adjusted multilevel models, is illustrated. Whiskers represent $95 \% \mathrm{Cl}$. ${ }^{\star}$ Significant difference, $p<0.05$; ${ }^{*}$ significant difference, $p<0.01$. JSN, joint space narrowing; JSW, joint space width.

joints are highly inter-related. The unadjusted multilevel model, however, had accounted for these factors and showed a significant difference in JSW between undamaged and damaged joints, $1.68 \mathrm{~mm}$ (95\% CI 1.72 to 1.64$)$ vs $1.63 \mathrm{~mm}$ ( $95 \%$ CI 1.68 to 1.58$)$ ( $\mathrm{p}=0.0048)$, and this difference remained significant after adjustment for height, age, gender and BMI, $1.66 \mathrm{~mm}$ (95\% CI 1.70 to $1.61)$ vs $1.62 \mathrm{~mm}$ (95\% CI 1.68 to 1.56$)(\mathrm{p}=0.042)$, respectively.

\section{DISCUSSION}

The aims of this study were to explore and evaluate the changes in JSW and BMD at baseline and 1-year follow-up compared with the JSN component in the SHS. This program is a further development of the program used by Böttcher $e t a l,{ }^{21}$ which was a semiautomated program that had an operator-dependent stage of evaluation. The non-dominant hand radiograph was used in the above study, whereas bilateral images of patients with early RA were used in our study. We also incorporated both BMD and JSW using the updated, operator-independent, fully automated program. This operator independence makes it more clinically viable as a diagnostic tool as you introduce less potential operator errors, minimise the time of data acquisition and avoid the training of technicians.

The fully automated method revealed a JSW and BMD reduction between baseline and 1-year follow-up. Significant differences in the JSW between damaged and non-damaged joints as rated by the JSN score were noted. The multilevel mixed model also showed a significant positive association between $\triangle \mathrm{BMD}$ and $\triangle \mathrm{JSW}$ from baseline to 1-year follow-up.

It is known that one of the markers of radiographic progression in RA is cartilage damage, displayed as narrowing of the JSW. ${ }^{2627}$ Since effective disease-modifying antirheumatic drug (DMARD) treatments may slow down radiographic progression, it is important to be able to detect subtle changes, especially in the early stages of RA, to improve treatment in order to achieve improved radiographic and clinical outcome. ${ }^{28} \mathrm{~A}$ sensitive and objective method to detect cartilage loss is valuable, but it also needs to be reproducible. The reproducibility of the fully automated JSW was evaluated with three repeated radiographs of the non-dominant hand of 30 healthy volunteers and resulted in a CV\% of $1.4 \%$ and an SDD of $0.062 \mathrm{~mm}$. This result is similar to the results presented with the semiautomated program by Pfeil et al. ${ }^{13}$ High sensitivity and reproducibility are a major strength of automated JSW seen in several studies. ${ }^{72}{ }^{23}$ In our study, we illustrate that with this automated method, we are able to detect a difference in JSW between damaged and undamaged joints, as defined according to the JSN component of the validated SHS method. Furthermore, the difference in JSW was significant in the unadjusted mixed model and remained significant after adjustment for height, age, gender and BMI, which have previously been shown to be confounding variables for measurements of JSW in RA. ${ }^{22} 232529$ This suggests that the loss of cartilage as shown by this fully automated JSW measuring method may primarily be due to the progression of the actual disease. Another strength of a fully automated JSW measurement is that it may help remedy the limitations of measuring JSN by SHS or by visual inspection alone. Limitations such as inter-reader and intrareader variations and the necessity of qualified readers are thus eliminated. ${ }^{30}$ Also important in this study is the need for high-quality acquisition radiographs that are free from technical and positional errors. This for us is a strength rather than a weakness, from the perspective of establishing radiographic quality control and assurance of good radiological practice.

Rapid loss of BMD may take place in the early stages of RA and predicts future radiographic progression as assessed with SHS. ${ }^{16-19}$ Compared with CR, which reportedly only detects damage if the reduction in bone density is more than $35 \%,{ }^{16} 1731$ digital BMD measuring has a higher sensitivity. This may be important in the clinic when DMARDs may halt or slow down progression to a point where the CR method is not sensitive enough to measure a difference. Digital BMD measurement, however, is a sensitive tool that has been shown to distinguish treatment effects. ${ }^{15}$ To measure BMD is an objective and quick method for quantifying bone loss. ${ }^{32}$ In our study, BMD decreased significantly in both hands during 12 months, suggesting a progression of damage. A significant positive association was observed in the multilevel model between $\triangle \mathrm{BMD}$ and $\triangle \mathrm{JSW}$. This suggests that the loss of BMD and JSW, that is, bone and cartilage loss, are two processes that are occurring concurrently in RA.

In a clinical setting, it is important to have a very low failure rate of image analysis so as to not impede the workflow. In our study the failure rate was $0 \%$ for the analysis of six MCP joints per patient - following the 
inclusion criteria. It is important that the analysis is not too time-consuming, in which case it would outweigh its benefit. The time to score seven radiographs of the hands and feet has been documented to take $19 \mathrm{~min}$ for the Sharp method and $25 \mathrm{~min}$ for the modified SHS method. ${ }^{1}$ A fully automated software method may decrease the time spent assessing radiographs as well as increase the accuracy of subtle joint space reductions.

Limitations of this study include its design as a post-hoc analysis from the SWEFOT trial, which was not primarily intended for evaluation of JSW or BMD. This resulted in the inclusion of 119 of 487 patients from the original SWEFOT study, of whom only 96 had both baseline and follow-up digital radiographs useful for our evaluation. There were also relatively few patients with progression of joint damage as measured by SHS, since this was a trial with patients with early RA. This however highlighted the utility of a highly sensitive method such as the one used in this study for monitoring early cartilage damage in RA. Another limitation of the study includes the absence of JSW of MCP 1 and 5. The JSW of the proximal interphalangeal (PIP) II-IV joints were available but omitted due to the association of these joints to osteoarthritis (OA). Furthermore, the MCP joints 2 and 3 have a stronger relationship to RA than the PIP joints, which are more prone to be affected by OA. In addition, the PIP joints are also more complicated as they have a bicompartmental configuration, which makes them very sensitive to minor rotations of the hand. Moreover, the unknown characteristics of JSW in RA as the disease progresses through various stages require further investigation. For example, bisphosphonates have shown to prevent generalised bone loss. Their main target is the osteoclast, identified as a potential culprit of focal bone damage caused by inflammatory diseases. The potential effects of bisphosphonates in focal bone damage related to RA are certainly of great interest, and although we have not tested this it may be beneficial in future RA studies to compare BMD and JSW results on patients who are on bisphosphonates compared with those who are not. Although normative values for JSW have been reported previously, ${ }^{33}$ further studies on normal cartilage degeneration over time are warranted. Comparative US studies on cartilage damage and bone erosions may also add value to early RA assessments. Future studies to evaluate the utility of JSW could also be done together with the analysis of biomarkers, as biomarkers have shown to predict radiographic progression as measured by SHS. ${ }^{34}$

In summary, to measure the JSW with this fully automated digital program may be useful as a quick and observer-independent tool for evaluating cartilage damage in early RA. We have shown that a fully automated JSW measurement method was associated with the JSN component of the SHS method, and that it had the further clinical feasibility of being quick and observer-independent. We also showed a significant positive association between $\triangle \mathrm{BMD}$ and $\triangle \mathrm{JSW}$ from baseline to 1-year follow-up. Fully automated measuring of JSW with the added benefit of BMD makes JSW measuring a potentially useful clinical tool.

Author affiliations

${ }^{1}$ Department of Medicine, Unit for Clinical Therapy Research, Inflammatory

Diseases (ClinTRID), Karolinska Institute, Stockholm, Sweden

${ }^{2}$ Medicine and Health Sciences, Center for Medical Image Science and Visualization (CMIV), Linköping University, Linköping, Sweden

${ }^{3}$ Sectra AB, Linköping, Sweden

${ }^{4}$ Department of Medicine, Section of Rheumatology, Helsingborg's Hospital, Helsingborg, Sweden

${ }^{5}$ Department of Clinical Sciences, Section of Rheumatology, Lund University, Helsingborg, Sweden

${ }^{6}$ Departments of AMC, READE and VUmc, Amsterdam Rheumatology \& Immunology Center (ARC), Amsterdam, Netherlands

Acknowledgements We wish to thank all the participating clinicians in the SWEFOT study group, nurses and patients who made the original trial possible.

Contributors MP led the writing of the manuscript, analysed data and interpreted results. YK participated in statistical analyses and interpretation of the results. JK was responsible for JSW measuring sampling and data recovery. LA participated in the statistical analyses and interpretation of the results. KF was responsible for the radiographic assessments including the scoring of radiographs. RvV inspired the study, designed it and contributed to analysis. All authors were involved in drafting the article or revising it critically for important intellectual content, and all authors approved the final version to be submitted for publication. MP had full access to all of the data in the study and takes responsibility for the integrity of the data and the accuracy of the data analysis.

Funding This work was supported by grants from the Swedish Rheumatism Association, the Foundation for Assistance to Disabled People in Skåne (Stiftelsen för bistånd åt Rörelsehindrade i Skåne), Stockholm County, Schering-Plough/Merck Sharp and Dohme.

Competing interests $\mathrm{JK}$ is employed by Sectra AB. No other conflicts of interests to declare.

Ethics approval All patients gave their informed consent and the study was performed in accordance with the Declaration of Helsinki. Ethical approval for the present study was obtained from the ethical board in all participating units and was registered (NCT00764725).

Provenance and peer review Not commissioned; externally peer reviewed.

Data sharing statement Access to raw study data can be granted on contact with the main author.

Open Access This is an Open Access article distributed in accordance with the Creative Commons Attribution Non Commercial (CC BY-NC 4.0) license, which permits others to distribute, remix, adapt, build upon this work non-commercially, and license their derivative works on different terms, provided the original work is properly cited and the use is non-commercial. See: http://creativecommons.org/ licenses/by-nc/4.0/

(c) Article author(s) (or their employer(s) unless otherwise stated in the text of the article) 2017. All rights reserved. No commercial use is permitted unless otherwise expressly granted.

\section{REFERENCES}

1. Boini S, Guillemin F. Radiographic scoring methods as outcome measures in rheumatoid arthritis: properties and advantages. Ann Rheum Dis 2001;60:817-27.

2. McQueen FM. Imaging in early rheumatoid arthritis. Best Pract Res Clin Rheumatol 2013;27:499-522.

3. Lillegraven S, van der Heijde D, Uhlig T, et al. What is the clinical relevance of erosions and joint space narrowing in RA? Nat Rev Rheumatol 2012;8:117-20.

4. van der Heijde D. How to read radiographs according to the sharp/ van der Heijde method. J Rheumatol 2000;27:261-3.

5. Aletaha D, Funovits J, Smolen JS. Physical disability in rheumatoid arthritis is associated with cartilage damage rather than bone destruction. Ann Rheum Dis 2011;70:733-9.

6. Sharp JT, Gardner JC, Bennett EM. Computer-based methods for measuring joint space and estimating erosion volume in the finger and wrist joints of patients with rheumatoid arthritis. Arthritis Rheum 2000;43:1378-86. 
7. Angwin J, Lloyd A, Heald G, et al. Radiographic hand joint space width assessed by computer is a sensitive measure of change in early rheumatoid arthritis. J Rheumatol 2004;31:1050-61.

8. Sharp JT, van der Heijde D, Angwin J, et al. Measurement of joint space width and erosion size. J Rheumatol 2005;32:2456-61.

9. Huo Y, Vincken KL, van der Heijde D, et al. Automatic quantification of Radiographic Finger Joint Space Width of Patients with Early Rheumatoid Arthritis. IEEE Trans Biomed Eng 2016;63:2177-86.

10. Schenk O, Huo Y, Vincken KL, et al. Validation of automatic joint space width measurements in hand radiographs in rheumatoid arthritis. J Med Imaging 2016;3:044502.

11. Lukas C, Sharp JT, Angwin J, et al. Automated measurement of joint space width in small joints of patients with rheumatoid arthritis. $J$ Rheumatol 2008;35:1288-93

12. Neumann $\mathrm{G}$, dePablo $\mathrm{P}$, Finckh $\mathrm{A}$, et al. Patient repositioning reproducibility of joint space width measurements on hand radiographs. Arthritis Care Res 2011;63:203-7.

13. Pfeil A, Hansch A, Sommerfeld J, et al. Reproducibility and influence of hand rotation on computer-aided joint space analysis. Joint Bone Spine 2012;79:384-8.

14. Pfeil A, Haugeberg G, Hansch A, et al. Value of digital X-ray radiogrammetry in the assessment of inflammatory bone loss in rheumatoid arthritis. Arthritis Care Res 2011;63:666-74.

15. Pfeil A, Oelzner P, Renz DM, et al. Is there a role for Digital X-ray radiogrammetry as surrogate marker for radiological progression and imaging of structural integrity in rheumatoid arthritis? BMC Musculoskelet Disord 2015;16:155.

16. Rezaei H, Saevarsdottir S, Geborek P, et al. Evaluation of hand bone loss by digital X-ray radiogrammetry as a complement to clinical and radiographic assessment in early rheumatoid arthritis: results from the SWEFOT trial. BMC Musculoskelet Disord 2013;14:79.

17. Forslind K, Boonen A, Albertsson K, et al. Hand bone loss measured by digital $X$-ray radiogrammetry is a predictor of joint damage in early rheumatoid arthritis. Scand J Rheumatol 2009;38:431-8.

18. Rastogi A, Algulin J, Mangat $P$, et al. Early metacarpal bone mineral density loss using digital $\mathrm{X}$-ray radiogrammetry and 3-tesla wrist MRI in established rheumatoid arthritis: a longitudinal one-year observational study. Arthritis 2015;2015:852989-.

19. Wevers-de Boer KV, Heimans L, Visser K, et al. Four-month metacarpal bone mineral density loss predicts radiological joint damage progression after 1 year in patients with early rheumatoid arthritis: exploratory analyses from the IMPROVED study. Ann Rheum Dis 2015;74:341-6.

20. van Vollenhoven RF, Ernestam S, Geborek P, et al. Addition of infliximab compared with addition of sulfasalazine and hydroxychloroquine to methotrexate in patients with early rheumatoid arthritis (Swefot trial): 1-year results of a randomised trial. Lancet 2009;374:459-66.

21. Böttcher J, Pfeil A, Rosholm A, et al. Digital X-ray radiogrammetry combined with semiautomated analysis of joint space widths as a new diagnostic approach in rheumatoid arthritis: a cross-sectional and longitudinal study. Arthritis Rheum 2005;52:3850-9.

22. Pfeil A, Böttcher J, Seidl BE, et al. Computer-aided joint space analysis (CAJSA) of the proximal-interphalangeal jointnormative age-related and gender specific data. Acad Radiol 2007;14:594-602.

23. Pfeil A, Renz DM, Hansch A, et al. The usefulness of computer-aided joint space analysis in the assessment of rheumatoid arthritis. Joint Bone Spine 2013;80:380-5.

24. Rosholm A, Hyldstrup L, Backsgaard L, et al. Estimation of bone mineral density by digital $\mathrm{X}$-ray radiogrammetry: theoretical background and clinical testing. Osteoporos Int 2001;12:961-9.

25. Pfeil A, Hansch A, Lehmann G, et al. Impact of sex, age, body mass index and handedness on finger joint space width in patients with prolonged rheumatoid arthritis using computer-aided joint space analysis. Rheumatol Int 2009;29:517-24.

26. Burghardt AJ, Lee $\mathrm{CH}$, Kuo D, et al. Quantitative in vivo HR-pQCT imaging of 3D wrist and metacarpophalangeal joint space width in rheumatoid arthritis. Ann Biomed Eng 2013;41:2553-64.

27. Snekhalatha $U$, Anburajan M. Computer-based measurements of joint space analysis at metacarpal morphometry in hand radiograph for evaluation of rheumatoid arthritis. Int J Rheum Dis 2015:n/a (published Online First: 2015/04/14).

28. Huo Y, De Hair MJ, Shaib YO, et al. Computerised versus conventional methodology of radiographic joint destruction assessment in early rheumatoid arthritis. RMD Open 2015;1:e000148.

29. Goligher EC, Duryea J, Liang MH, et al. Radiographic joint space width in the fingers of patients with rheumatoid arthritis of less than one year's duration. Arthritis Rheum 2006;54:1440-3.

30. Peloschek P, Langs $\mathrm{G}$, Weber M, et al. An automatic model-based system for joint space measurements on hand radiographs: initial experience. Radiology 2007;245:855-62.

31. Jergas $\mathrm{M}$, Uffmann $\mathrm{M}$, Escher $\mathrm{H}$, et al. Interobserver variation in the detection of osteopenia by radiography and comparison with dual X-ray absorptiometry of the lumbar spine. Skeletal Radiol 1994;23:195-9.

32. Forsblad-d'Elia $\mathrm{H}$, Carlsten $\mathrm{H}$. Bone mineral density by digital X-ray radiogrammetry is strongly decreased and associated with joint destruction in long-standing rheumatoid arthritis: a cross-sectional study. BMC Musculoskelet Disord 2011;12:242.

33. Pfeil A, Böttcher J, Seidl BE, et al. Computer-aided joint space analysis of the metacarpal-phalangeal and proximal-interphalangeal finger joint: normative age-related and gender-specific data. Skeletal Radiol 2007;36:853-64.

34. Hambardzumyan K, Bolce R, Saevarsdottir S, et al. Pretreatment multi-biomarker disease activity score and radiographic progression in early RA: results from the SWEFOT trial. Ann Rheum Dis 2015;74:1102-9. 